\title{
Dysregulated genes targeted by microRNAs and metabolic pathways in bladder cancer revealed by bioinformatics methods
}

\author{
LU ZHANG $^{1 *}$, CUIHUA FENG $^{2 *}$, YAMIN ZHOU $^{3}$ and QIONG ZHOU ${ }^{1}$ \\ Departments of ${ }^{1}$ Urology and ${ }^{2}$ Gastrointestinal Surgery; ${ }^{3}$ Intensive Care Unit, \\ Wuhan No. 6 Hospital, Wuhan, Hubei 430015, P.R. China
}

Received August 19, 2016; Accepted September 28, 2017

DOI: $10.3892 /$ ol.2018.8602

\begin{abstract}
The present study aimed to identify bladder cancer-associated microRNAs (miRNAs) and target genes, and further analyze the potential molecular mechanisms involved in bladder cancer. The mRNA and miRNA expression profiling dataset GSE40355 was downloaded from the Gene Expression Omnibus database. The Limma package in $\mathrm{R}$ was used to identify differential expression levels. The Human microRNA Disease Database was used to identify bladder cancer-associated miRNAs and Target prediction programs were used to screen for miRNA target genes. Enrichment analysis was performed to identify biological functions. The Database for Annotation, Visualization and Integration Discovery was used to perform OMIM_DISEASE analysis, and then protein-protein interaction (PPI) analysis was performed to identify hubs with biological essentiality. ClusterONE plugins in cytoscape were used to screen modules and the InterPro database was used to perform protein domain enrichment analysis. A group of 573 disease dysregulated genes were identified in the present study. Enrichment analysis indicated that the muscle organ development and vascular
\end{abstract}

Correspondence to: Dr Yamin Zhou, Intensive Care Unit, Wuhan No. 6 Hospital, 168 Hongkong Road, Wuhan, Hubei 430015, P.R. China

E-mail: yaminzhou@aliyun.com

Dr Qiong Zhou, Department of Urology, Wuhan No. 6 Hospital, 168 Hongkong Road, Wuhan, Hubei 430015, P.R. China

E-mail: 596624661@qq.com

*Contributed equally

Abbreviations: HMDD, Human microRNA Disease Database; DAVID, Database for Annotation, Visualization and Integration Discovery; PPI, protein-protein interaction; KEGG, Kyoto Encyclopedia of Genes and Genomes; GO, Gene Ontology; GEO, Gene Expression Omnibus; FDR, false discovery rate; FC, fold-change; DEGs, differentially expressed genes; STRING, Search Tool for the Retrieval of Interacting Genes; CALM, calmodulin

Key words: bladder cancer, microRNA targets, disease dysregulated genes, bioinformatics methods smooth muscle contraction pathways were significantly enriched in terms of disease dysregulated genes. miRNAs targets (frizzled class receptor 8, EYA transcriptional coactivator and phosphatase 4 , sacsin molecular chaperone, calcium voltage-gated channel auxiliary subunit $\beta 2$, peptidase inhibitor 15 and catenin $\alpha 2$ ) were mostly associated with bladder cancer. PPI analysis revealed that calmodulin 1 (CALM1), Jun proto-oncogene, AP-1 transcription factor subunit (JUN) and insulin like growth factor 1 (IGF1) were the important hub nodes. Additionally, protein domain enrichment analysis indicated that the serine/threonine protein kinase active site was enriched in module 1 extracted from the PPI network. Overall, the results suggested that the IGF signaling pathway and RAS/MEK/extracellular signal-regulated kinase transduction signaling may exert vital molecular mechanisms in bladder cancer, and that CALM1, JUN and IGF1 may be used as novel potential therapeutic targets.

\section{Introduction}

Bladder cancer is reported to be one of the four most commonly occurring genitourinary tumors in China (1), and is one of the most expensive types of cancer to treat due to the essential life-long surveillance involving upper tract imaging, urinary cytology and cystoscopy (2). The disease usually presents in two distinct forms: Non-muscle-invasive tumors in clinical stages Ta and T1 and muscle-invasive cancer occurring in clinical stages T2-4 (3). As bladder cancer may result from numerous processes with accumulation of genetic and epigenetic changes, high-throughput technologies have been used to generate numerous genetic and genomic datasets to uncover disease causal genes and their actions involved in the initiation and development of bladder cancer (4,5). A number of studies have documented a strong link between microRNA (miRNA/miR) function and cancer pathogenesis, including bladder cancer (6-8).

miRNAs represent a class of naturally non-coding RNAs (21-23 nucleotides in length), which act as post-transcriptional silencing modulators of target genes either by negatively regulating specific target mRNAs or by inhibiting target protein synthesis $(9,10)$. A previous study reported a potential diagnostic role of miRNAs, as the miRNAs-profiling revealed a more accurate diagnostic effect compared with the mRNAs-classifiers (7). miRNAs have received increasing attention in cancer genomic research and the novel oncogenic or tumor suppression functions 
of miRNAs have been revealed in a clinical study (6). Certain studies have provided a detailed insight into regulatory networks involving bladder cancer by integrated analysis of miRNA and mRNA data (5,7). Altered miRNA expression levels have been reported in bladder cancer $(3,11)$; however, the target genes of these miRNAs have not yet been fully elucidated, particularly in bladder cancer, and further analysis is required to elucidate the subsequent processes.

Biological interpretation of large gene lists derived from microarray analysis may require researchers to select the most useful genes for further investigation. The Database for Annotation, Visualization and Integration Discovery (DAVID) has emerged as a publicly available high-throughput functional annotation tool (12). Kyoto Encyclopedia of Genes and Genomes (KEGG) pathway enrichment analysis (13) and Gene Ontology (GO) term analysis (14) are able to identify the main functional and metabolic signaling pathways of differentially expressed genes (DEGs). The present study used KEGG pathways (http://www.genome.jp/kegg/pathway.html) and GO term (http://geneontology.org/) analysis for enrichment analysis.

In addition, genes with similar functions have been reported to interact with each other closely, as presented in the protein-protein interaction (PPI) network, which provides a global picture used to understand molecular mechanisms and biological processes of diseases, in particular to analyze cancer $(15,16)$. The PPI network has been used to systematically analyze and compare the disease genes that would otherwise not be identified by single gene analysis (17), in order to gain insights into distinct topological features of cancer genes using cancer-associated sub-networks. Hub nodes are known to be the most important nodes in the PPI network, as the corresponding proteins are important proteins in metabolic networks. In the present study, disease dysregulated genes were mapped into the PPI network, where proteins are represented as nodes and interactions as edges, in order to understand key biological processes involved in bladder cancer in a global sense.

miRNAs exert their function through translational repression or degradation of mRNA targets, and of particular note is the fact that no study has yet systematically analyzed the significant mechanisms underlying miRNA targets contributing to bladder cancer incidence. In the present study, numerous computational methods were used to analyze microarray data of miRNAs and mRNAs between bladder cancer and normal samples, in an attempt to describe the variation of miRNA targets and the potential mechanisms involved in the development of bladder cancer.

\section{Materials and methods}

miRNA and mRNA expression profiling data. Gene Expression Omnibus (GEO; http://www.ncbi.nlm.nih.gov/geo/), which is established at the National Center for Biotechnology Information, provides high-throughput gene expression data from the research community worldwide. The microarray expression profiling data of miRNAs and mRNAs in bladder urothelial carcinoma ('case') and normal bladder tissue samples ('normal') were downloaded from the GEO database (dataset GSE40355). Detailed messages were accessible as follows: Microarray data of miRNAs included
8 normal (GSM881439-GSM881446) and 8 case chips (GSM881455-GSM881462); and microarray data of mRNAs included 8 normal (GSM991923-GSM991930) and 8 case chips (GSM991939-GSM991946). The expression levels of miRNAs and mRNAs were previously studied in these samples on two platforms: GPL8227 Agilent-019118 Human miRNA Microarray 2.0 G4470B (miRNA ID version) and GPL13497 Agilent-026652 Whole Human Genome Microarray 4x44K v2 (Probe Name version) (both Agilent Technologies, Inc., Santa Clara, CA, USA) (18).

Identification of differentially expressed miRNAs and genes. The raw microarray expression data of miRNAs and mRNAs downloaded as Series Matrix files from the GEO database were mapped to the corresponding genes according to the SOFT formatted family files from GEO database. For each sample, the expression values of all probes for the same miRNA or mRNA were reduced to a single value by calculating the average expression value. Bayesian method was used for multiple comparisons under the control of false discovery rate (FDR) (19). Limma package in R software (version 3.24.15, https://www.bioconductor.org/packages/3.1/bioc/html/limma.html; Bioconductor; Fred Hutchinson Cancer Reearch Center, Seattle, WA, USA) (20), a linear regression model, was used to identify differentially expressed miRNAs and genes with the cut-off criteria of $\mid \log$ fold change (FC) $\mid>1, \mathrm{P}<0.05$ and FDR $<0.01$ (21).

Identification of disease dysregulated genes. To identify the disease miRNAs associated with bladder cancer, the selected differentially expressed miRNAs were mapped to the online software, Human microRNA Disease Database (HMDD; version 2.0; 2014.06.14 update; http://www.cuilab.cn/hmdd). As the functions of miRNAs were dependent on its target genes (9), the target genes of disease miRNAs were screened using target prediction programs. A single miRNA can regulate numerous mRNAs and a single mRNA may be targeted by different miRNAs. In order to ensure a high level of accuracy, five target prediction programs, including miRanda http://www.microrna.org), MirTarget2 (http://mirdb.org/miRDB), PicTar (http://www.pictar.org/), PITA (http://genie.weizmann.ac.il/pubs/mir07/mir07_ data.html) and TargetScan (http://www.targetscan.org/) were used to screen potential targets of miRNAs (22). To decrease the number of false-positive results, putative target genes predicted by at least 3 programs of the five were accepted as the disease miRNAs targets.

Finally, to identify which differentially expressed miRNAs targets were dysregulated in bladder cancer, the present study selected the intersection of disease miRNA targets and DEGs via the mRNAs chip data obtained above. The common genes were considered as the disease dysregulated genes for further analysis.

Enrichment analysis and cancer-associated gene screening. To further understand gene functions and identifying pathways closely associated with disease dysregulated genes, the functional annotation of associated genes was performed using the online software, DAVID. The present study used KEGG pathway and GO term analysis for the following enrichment analysis of disease dysregulated genes to identify 
Table I. Top five enriched GO terms.

\begin{tabular}{|c|c|c|c|}
\hline Category & Term & Count & P-value \\
\hline GOTERM_BP_FAT & GO:0007517 muscle organ development & 34 & $3.83 \times 10^{-14}$ \\
\hline GOTERM_BP_FAT & GO:0042692 muscle cell differentiation & 22 & $2.54 \times 10^{-10}$ \\
\hline GOTERM_BP_FAT & GO:0014706 striated muscle tissue development & 21 & $1.23 \times 10^{-9}$ \\
\hline GOTERM_BP_FAT & GO:0060537 muscle tissue development & 21 & $3.00 \times 10^{-9}$ \\
\hline GOTERM_BP_FAT & GO:0007167 enzyme-linked receptor protein signaling pathway & 35 & $5.26 \times 10^{-9}$ \\
\hline
\end{tabular}

Count represents the number of genes which were enriched in the corresponding functional category. GO,Gene Ontology; GOTERM_BP_FAT, Gene Ontology term-biological process.

the function and pathway alterations involved in bladder cancer, with $\mathrm{P}<0.05$ used as the cut-off criterion. Furthermore, DAVID was used to perform OMIM_DISEASE analysis, in order to distinguish cancer-associated genes from the disease dysregulated genes.

PPI network and miRNA-gene regulatory network. Highly complex cellular functions are the results of tightly regulated interactions of groups of proteins encoded by genes. Interactions between proteins possess a crucial role in modern biology, thus a rapidly growing number of technologies have been developed for the global charting of the PPI network. The Search Tool for the Retrieval of Interacting Genes (STRING), an online database resource (http://string-db.org/), provides uniquely comprehensive information for assembling, evaluating and disseminating PPI in a user-friendly manner (23).

In the present study, STRING was used to screen the PPI of disease dysregulated genes, which was then visualized using Cytoscape software (http://cytoscape.org/; version 3.2.1; National Institute of General Medical Sciences, Seattle, WA, USA). The total connectivity degree of each node in the network was calculated using Cytoscape software and only the nodes with $\geq 30$ degrees were selected as the important nodes (Hubs) of PPI. Additionally, the integrated miRNA-disease dysregulated genes regulatory network was also constructed using Cytoscape software, followed by network module screening using the threshold of $\mathrm{P}<0.05$ by employing the clusterONE plugins in Cytoscape (24). Subsequently, protein domain enrichment analysis was performed on the screened modules using the InterPro database (25).

\section{Results}

Differentially expressed clustered miRNAs and genes. Based on the Limma package in R language, under cut-off criteria of $\mid \log \mathrm{FCl}>1, \mathrm{P}<0.05$ and $\mathrm{FDR}<0.01,112$ miRNAs and 2,290 genes were identified to be differentially expressed in bladder cancer samples compared with the control groups. There were 56 upregulated and 56 downregulated miRNAs; as well as 1,444 upregulated and 846 downregulated DEGs.

Target screening of the differentially expressed miRNAs. Firstly, differentially expressed miRNAs associated with bladder cancer were screened from the 112 miRNAs using the HMDD database and 71 bladder cancer-associated miRNAs were obtained from this procedure, including hsa-miR-143, hsa-miR-205, hsa-miR-21, hsa-miR-93, hsa-miR-145 and hsa-miR-143. Subsequently, targets of the 71 disease miRNAs were identified using five target prediction programs, including miRanda, MirTarget2, PicTar, PITA and Target. For each target selection, the present study selected the target genes of miRNAs coexisting in at least three of the databases.

Identification of disease dysregulated genes. A total of 573 disease dysregulated genes associated with bladder cancer were identified by taking the intersection of disease miRNA targets and 2,290 differentially expressed genes corresponding to mRNA datasets.

Enrichment analysis of disease dysregulated genes. To analyze the biological functions of disease dysregulated genes in bladder cancer, GO and KEGG pathway enrichment analyses were performed and the results are presented in Tables I and II, respectively. Following combination of the results from the GO and KEGG pathway analyses, it was revealed that the most marked function and pathway involved in bladder cancer were muscle organ development and vascular smooth muscle contraction, respectively. Furthermore, DAVID was used to perform OMIM_DISEASE analysis, and a set of six target genes [frizzled class receptor 8 (FZD8), sacsin molecular chaperone (SACS), calcium voltage-gated channel auxiliary subunit $\beta 2$ (CACNB2), peptidase inhibitor 15 (PI15) and catenin $\alpha 2$ (CTNNA2)] were identified to closely associate with the functional term of disease of cancer, suggesting a potential association with bladder cancer.

PPI network and integrated miRNA-target network construction. In order to gain further insights into the changes of biological pathways in bladder cancer, the present study used Cytoscape to identify the PPI of disease dysregulated genes (Fig. 1). The upregulated calmodulin 1 (CALM1), jun proto-oncogene (JUN) and insulin-like factor 1 (IGF1) were key hub nodes in the PPI network. Cytoscape was also used to perform regulatory network construction of disease dysregulated genes and the corresponding miRNAs, as shown in Fig. 2.

Module screening and subsequent protein domain enrichment analysis. ClusterONE plugins were used to perform module clustering analysis in the PPI network, with a criterion of 
Table II. Top five enriched KEGG pathways.

\begin{tabular}{llrr}
\hline Category & \multicolumn{1}{c}{ Term } & Count & P-value \\
\hline KEGG_PATHWAY & hsa04270: Vascular smooth muscle contraction & 15 & $2.45 \times 10^{-5}$ \\
KEGG_PATHWAY & hsa04360: Axon guidance & 15 & $1.19 \times 10^{-4}$ \\
KEGG_PATHWAY & hsa04510: Focal adhesion & 19 & $1.61 \times 10^{-4}$ \\
KEGG_PATHWAY & hsa04310: Wnt signaling pathway & 15 & $6.22 \times 10^{-4}$ \\
KEGG_PATHWAY & hsa04020: Calcium signaling pathway & 15 & $2.74 \times 10^{-3}$ \\
\hline
\end{tabular}

Count represents the number of genes which were enriched in the corresponding functional category. KEGG, Kyoto Encyclopedia of Genes and Genomes.

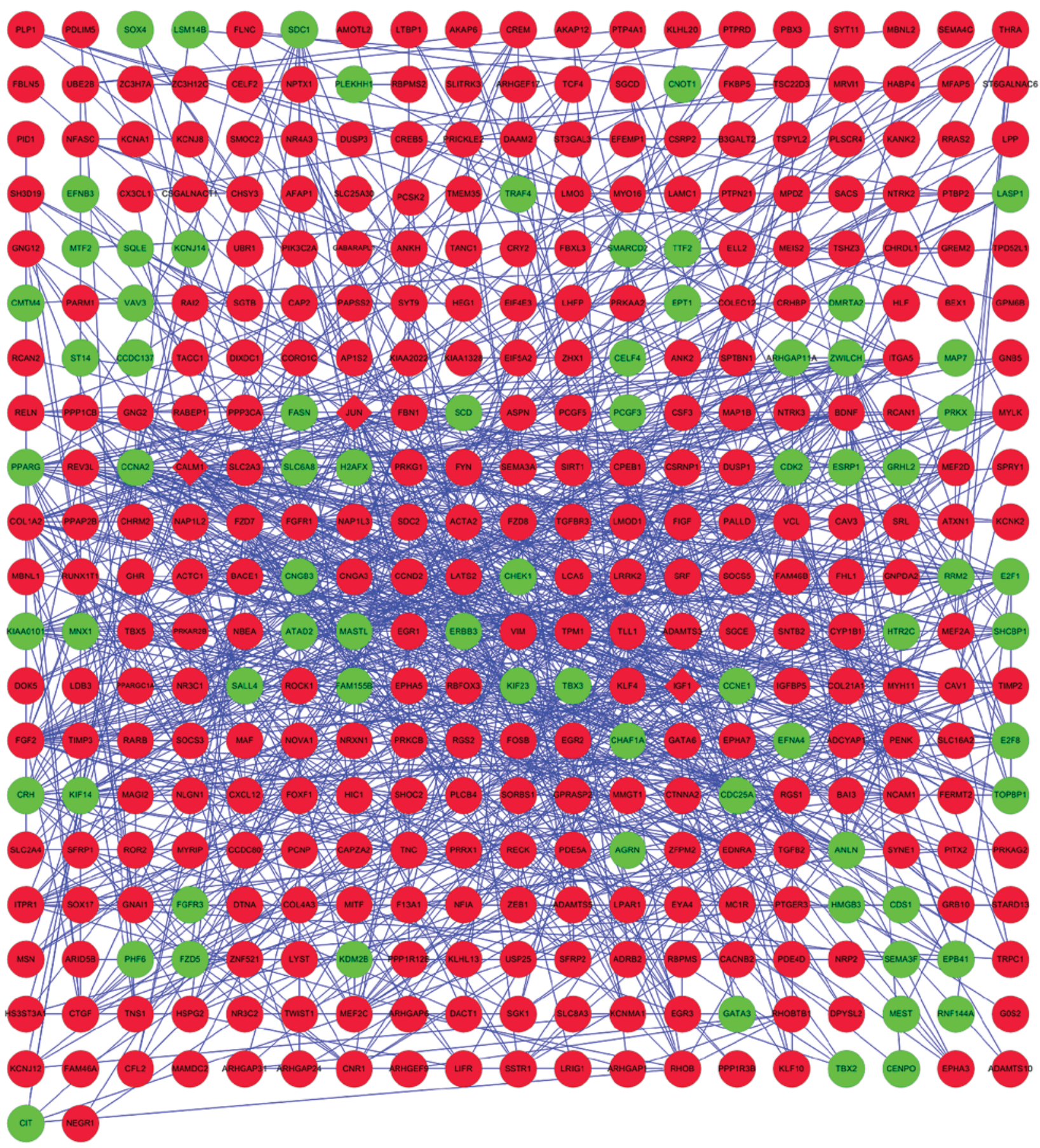

Figure 1. Protein-protein interaction network of disease dysregulated genes. The red nodes represent upregulated genes and the green nodes represent downregulated genes. Rhombuses represent hub nodes. 


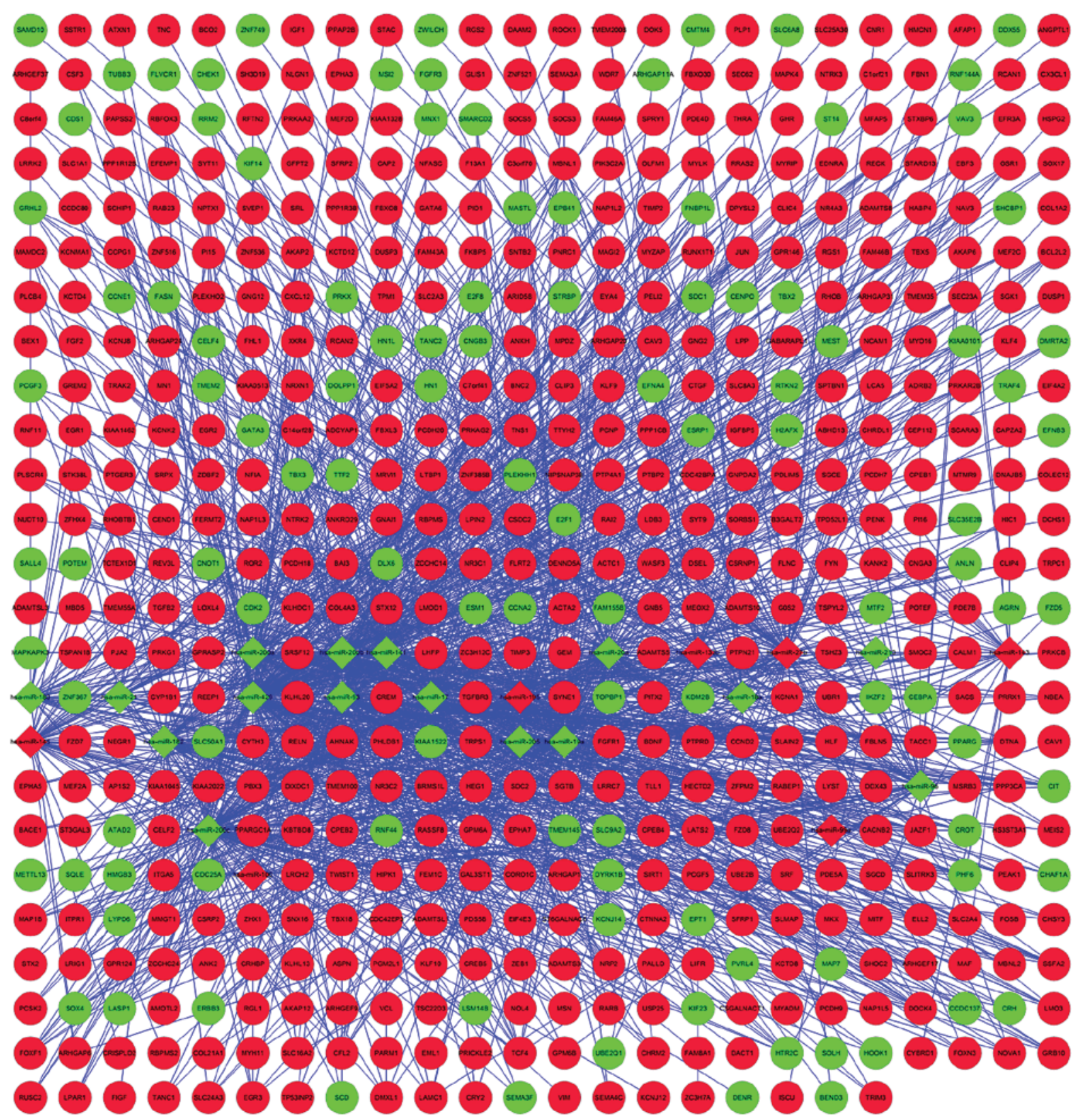

Figure 2. Integrated target miRNA interaction network. Red represents upregulation and green represents downregulation. Circles represent disease dysregulated genes and rhombuses represent corresponding miRNAs. miRNAs, microRNAs.

$\mathrm{P}<0.05$. A total of three clustering module were identified: Module 1 (density, 0.561 ; quality, $0.732 ; \mathrm{P}=1.582 \times 10^{-7}$ ), module 2 (density, 0.714 ; quality, $0.625 ; \mathrm{P}=0.002$ ) and module 3 (density, 0.526; quality, 0.512; $\mathrm{P}=0.004$ ) (Fig. 3). The protein domain enrichment analysis revealed that there were no significant enrichment results in module 2 and module 3 , whereas in module 1 the contained disease dysregulated genes, including SHC SH2-domain binding protein 1 (SHCBP1), were mainly enriched at IPR008271: Serine/threonine protein kinase active site (Table III).

\section{Discussion}

Recently, emerging bioinformatic methods have accelerated the progress made to identify the mechanisms involved in bladder cancer development at the molecular level. miRNAs were reported to regulate the activity of $\sim 30 \%$ of all protein-coding genes in the human genome (26), whereas the deregulation of miRNAs and their targeted mRNAs may serve an important function in the development of cancer. It was previously revealed that aberrantly expressed miRNAs served significant roles in the initiation, development and metastasis of bladder cancer $(3,27)$. Previous studies have identified the large set of differentially expressed miRNAs between bladder cancer and normal samples $(11,27,28)$. The present study further analyzed the expression profile data of miRNAs and mRNAs, and identified a total of 573 differentially expressed targets of the differentially expressed miRNAs in bladder cancer. These genes provided a global perspective for understanding the mechanism underlying bladder tumorigenesis. In addition, the results of functional enrichment analysis indicated that the genes were most significantly associated 


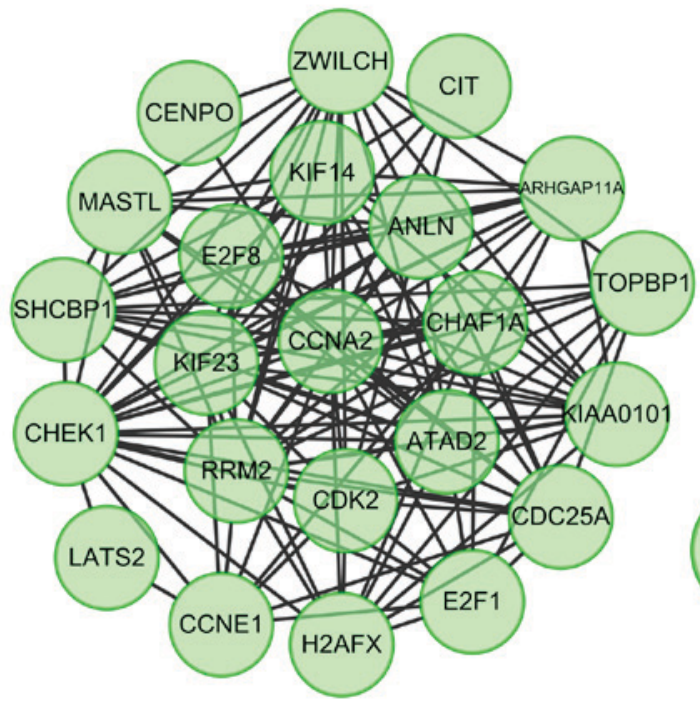

Module 1

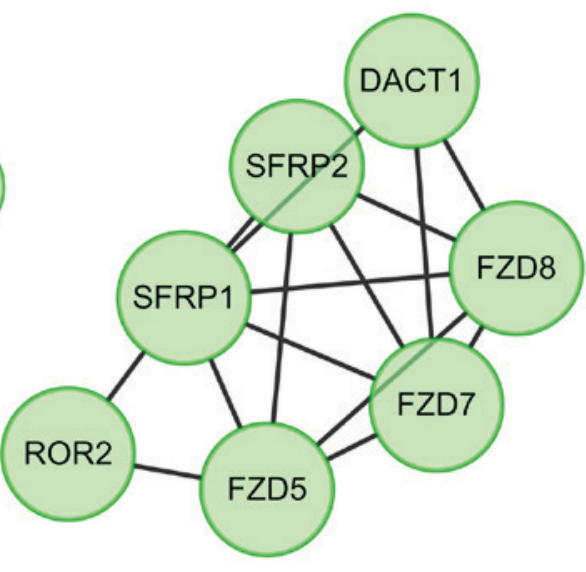

Module 2

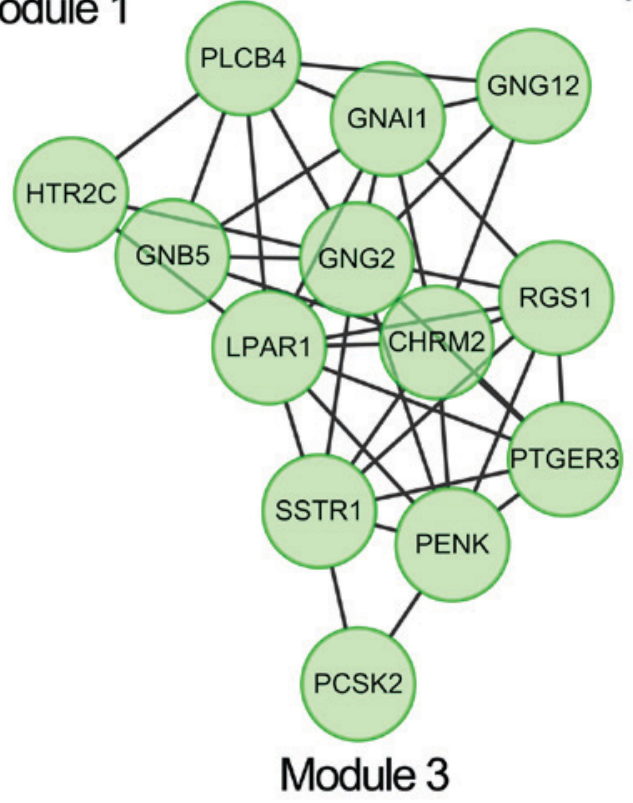

Figure 3. Corresponding clustering network modules extracted from the protein-protein interaction network.

with the GO term of muscle organ development and vascular smooth muscle contraction pathway. Genes, including FZD8, EYA transcriptional coactivator and phosphatase 4 (EYA4), SACS, CACNB2, PI15 and CTNNA2 in the PPI network, were demonstrated to be potentially associated with bladder cancer by OMIM_DISEASE analysis. Furthermore, CALM1, JUN and IGF1 were important hub nodes in the PPI network with high degrees of connectivity.

Previous evidence suggested that there were two distinct and overlapping stages of bladder cancer: Non-muscle-invasive and progressive muscle-invasive tumors (3). GO term and KEGG pathway enrichment analysis of disease dysregulated genes revealed that muscle organ development and vascular smooth muscle contraction, respectively, were involved in bladder cancer, which suggested a vital role of normal muscle function in preventing bladder cancer progression. On the molecular level, a previous study reported the participation of tumor-initiating cells, i.e., cancer stem cells, in urothelial
cancer(29).FZD8, EYA4, SACS, CACNB2, PI15 and CTNNA2 were demonstrated to be the cancer-associated target genes of differential miRNAs. Tumor suppression miRNAs targeting FZDs were reported to participate in the suppression of cell growth and metastasis via the Wnt signaling pathway, which served important roles in tumorigenesis (30). EYA4, PI15 and CTNNA2 functioned as tumor suppressors in the tumorigenesis of Barrett's esophageal cancer (31), glioblastoma (32) and laryngeal carcinomas (33), respectively. CACNB2 was downregulated in breast cancer cells, thereby causing poor signaling by negatively affecting the calcium metabolism (34). SACS targeted by miR-223 was reported to be upregulated in urothelial carcinoma in comparison to that in the normal mucosa (11). Notably, in the present study, the expression levels of 6 miRNA targets were revealed to be markedly increased in bladder cancer compared with those in the normal samples, which implied the potential involvement in bladder cancer development. 
Table III. Protein Domain enrichment analysis on module 1 using the InterPro database.

\begin{tabular}{llrl}
\hline Category & & \multicolumn{1}{c}{ Term } & Count \\
\hline INTERPRO & IPR008271: Serine/threonine protein kinase, active site & 5 & P-value \\
INTERPRO & IPR017442: Serine/threonine protein kinase-related & 5 & $7.42 \times 10^{-4}$ \\
INTERPRO & IPR017441: Protein kinase, ATP binding site & 5 & $7.82 \times 10^{-4}$ \\
INTERPRO & IPR000961: AGC-kinase, C-terminal & 3 & $1.88 \times 10^{-3}$ \\
INTERPRO & IPR000719: Protein kinase, core & 5 & $1.96 \times 10^{-3}$ \\
INTERPRO & IPR002290: Serine/threonine protein kinase & 4 & $2.22 \times 10^{-3}$ \\
INTERPRO & IPR015633: E2F Family & 2 & $3.48 \times 10^{-3}$ \\
INTERPRO & IPR003316: Transcription factor E2F/dimerization partner & 2 & $9.57 \times 10^{-3}$ \\
INTERPRO & IPR014400: Cyclin, A/B/D/E & 2 & $1.31 \times 10^{-2}$ \\
INTERPRO & IPR004367: Cyclin, C-terminal & 2 & $1.43 \times 10^{-2}$ \\
INTERPRO & IPR006671: Cyclin, N-terminal & 2 & $1.67 \times 10^{-2}$ \\
INTERPRO & IPR013763: Cyclin-related & 2 & $3.89 \times 10^{-2}$ \\
bINTERPRO & IPR006670: Cyclin & 2 & $4.35 \times 10^{-2}$ \\
INTERPRO & IPR017892: Protein kinase, C-terminal & 2 & $4.70 \times 10^{-2}$ \\
INTERPRO & IPR001752: Kinesin, motor region & 2 & $4.70 \times 10^{-2}$ \\
INTERPRO & IPR019821: Kinesin, motor region, conserved site & 2 & $4.93 \times 10^{-2}$ \\
\hline
\end{tabular}

Count represents the number of genes which were enriched in the corresponding functional category. ATP, adenosine triphosphate.

In order to further understand the oncogenic mechanisms underlying the screened miRNA targets involved in bladder cancer, the present study performed PPI analysis and identified three hub nodes: CALM1, JUN and IGF1. PPI networks are typically scale-free, in which hubs are 'highly connected' and tend to correspond to essential biological genes (35). IGF signaling, possessing an important effect on cell proliferation and differentiation, was implicated in numerous types of cancer and was thus considered to be a therapeutic target in cancer treatment (36). Dysregulated miRNAs targets were demonstrated to be involved in IGF1R signaling, as well as in its downstream antipoptotic signaling pathways, including phophoinisitide-3 kinase (PI3K)/protein kinase B (Akt)/mechanistic target of rapamycin (mTOR), in bladder urothelial carcinoma (37). Notably, the activation of the PI3K/Akt/mTOR signaling pathway was reported to be a candidate driver of the muscle-invasive phenotype of bladder cancer (38). In the present study, the upregulation of IGF1 suggested possible active IGF1R signaling and subsequent cascade dysregulation in bladder cancer. CALM, a ubiquitous $\mathrm{Ca}^{2+}$ receptor protein interacting with hundreds of different target proteins, revealed upregulated expression levels in numerous tumor cells in comparison with that in cells from normal tissues. CALM antagonists may inhibit tumor cells growth of different origins by affecting the CALM-dependent mechanisms (39). JUN, which is important for cell proliferation, survival and apoptosis, was reported to be a crucial contributing factor for tumorigenesis due to its overexpression in numerous types of human cancer (40). The results of the present study revealed that the increased expression levels of IGF1, CALM1 and JUN in bladder cancer may be potentially effective therapeutic targets for bladder cancer treatment.
In addition, the serine/threonine protein kinase active site was revealed to be enriched in genes contained in module 1 , which was extracted from the PPI network. The human protein kinase family, consisting of 518 genes, was classified as protein-serine/threonine kinases, protein-tyrosine kinases and tyrosine-kinase-like proteins based on the nature of the phosphorylated-hydroxy group $(41,42)$. The protein-serine/threonine kinases that participated in the RAS/RAF/MEK/extracellular signal-regulated kinase (ERK) signaling pathway were reported to be associated with retroviral oncogenes and were thereby attractive cancer drug targets (43-45). SHCBP1, a dysregulated gene in module 1, was previously demonstrated to regulate the expression of activated ERK1/2 and thus be necessary for the RAS/MEK/ERK signaling pathway (46). In the present study, the dysregulation of the serine/threonine protein kinase presented in module 1 may suggest abnormal RAS/MEK/ERK transduction signaling involved in bladder cancer progression.

In summary, the findings of the present study suggested the potential therapeutic ability of the IGF signaling pathway and RAS/MEK/ERK transduction signaling in bladder cancer, as well as bladder cancer-associated oncogenes, including FZD8, EYA4, SACS, CACNB2, PI15 and CTNNA2. Notably, CALM1, JUN and IGF1, which were identified as hubs in the PPI network, may be important therapeutic targets for bladder cancer intervention. However, further research, both epidemiological and mechanistic, is required to further validate the results and clarify the underlying molecular mechanisms.

\section{Acknowledgements}

The present study was supported by the Special Fund for Medical Service of Jilin Finance Department (grant no. SCZSY201507). 


\section{References}

1. Fangliu G and Yuli L: Changing status of genitourinary cancer in recent 50 years. Chin J Urol 2: 88-90, 2002.

2. Gaston KE and Grossman HB: Proteomic assays for the detection of urothelial cancer. Methods Mol Biol 641: 303-323, 2010.

3. Dyrskjøt L, Ostenfeld MS, Bramsen JB, Silahtaroglu AN, Lamy P, Ramanathan R, Fristrup N, Jensen JL, Andersen CL, Zieger K, et al: Genomic profiling of microRNAs in bladder cancer: miR-129 is associated with poor outcome and promotes cell death in vitro. Cancer Res 69: 4851-4860, 2009.

4. Dyrskjot L, Zieger K and Orntoft TF: Recent advances in high-throughput molecular marker identification for superficial and invasive bladder cancers. Front Biosci 12: 2063-2073, 2007.

5. Fendler A, Stephan C, Yousef GM and Jung K: MicroRNAs as regulators of signal transduction in urological tumors. Clin Chem 57: 954-968, 2011.

6. Wu W, Sun M, Zou GM and Chen J: MicroRNA and cancer: Current status and prospective. Int J Cancer 120: 953-960, 2007.

7. Li X, Chen J, Hu X, Huang Y, Li Z, Zhou L, Tian Z, Ma H, Wu Z, Chen M, et al: Comparative mRNA and microRNA expression profiling of three genitourinary cancers reveals common hallmarks and cancer-specific molecular events. PLoS One 6: e22570, 2011.

8. Guo AY, Sun J, Jia P and Zhao Z: A novel microRNA and transcription factor mediated regulatory network in schizophrenia. BMC Syst Biol 4: 10, 2010.

9. Bartel DP: MicroRNAs: Genomics, biogenesis, mechanism, and function. Cell 116: 281-297, 2004.

10. Ambros V: The functions of animal microRNAs. Nature 431: 350-355, 2004.

11. Gottardo F, Liu CG, Ferracin M, Calin GA, Fassan M, Bassi P, Sevignani C, Byrne D, Negrini M, Pagano F, et al: Micro-RNA profiling in kidney and bladder cancers. Urol Oncol 25: 387-392, 2007.

12. Huang da W, Sherman BT and Lempicki RA: Systematic and integrative analysis of large gene lists using DAVID bioinformatics resources. Nat Protoc 4: 44-57, 2009.

13. Kanehisa M and Goto S: KEGG: Kyoto encyclopedia of genes and genomes. Nucleic Acids Res 28: 27-30, 2000.

14. Harris MA, Clark J, Ireland A, Lomax J, Ashburner M, Foulger R, Eilbeck K, Lewis S, Marshall B, Mungall C, et al: The gene ontology (GO) database and informatics resource. Nucleic Acids Res 32 (Database Issue): D258-D261, 2004.

15. Kar G, Gursoy A and Keskin O: Human cancer protein-protein interaction network: A structural perspective. PLoS Comput Biol 5: e1000601, 2009.

16. Song J and Singh M: How and when should interactome-derived clusters be used to predict functional modules and protein function? Bioinformatics 25: 3143-3150, 2009.

17. Jia P, Kao CF, Kuo PH and Zhao Z: A comprehensive network and pathway analysis of candidate genes in major depressive disorder. BMC Syst Biol 5 (Suppl 3): S12, 2011.

18. Hecker N, Stephan C, Mollenkopf HJ, Jung K, Preissner R and Meyer HA: A new algorithm for integrated analysis of miRNA-mRNA interactions based on individual classification reveals insights into bladder cancer. PLoS One 8: e64543, 2013.

19. Efron B and Tibshirani R: Empirical bayes methods and false discovery rates for microarrays. Genet Epidemiol 23: 70-86, 2002.

20. Polpitiya AD, Qian WJ, Jaitly N, Petyuk VA, Adkins JN, Camp DG II, Anderson GA and Smith RD: DAnTE: A statistical tool for quantitative analysis of -omics data. Bioinformatics 24: 1556-1558, 2008.

21. Saito R, Smoot ME, Ono K, Ruscheinski J, Wang PL, Lotia S, Pico AR, Bader GD and Ideker T: A travel guide to Cytoscape plugins. Nat Methods 9: 1069-1076, 2012.

22. Xiao F, Zuo Z, Cai G, Kang S, Gao X and Li T: miRecords: An integrated resource for microRNA-target interactions. Nucleic Acids Res 37 (Database Issue): D105-D110, 2009.

23. Franceschini A, Szklarczyk D, Frankild S, Kuhn M, Simonovic M, Roth A, Lin J, Minguez P, Bork P, von Mering C and Jensen LJ: STRING v9. 1: Protein-protein interaction networks, with increased coverage and integration. Nucleic Acids Res 4137 (Database Issue): D808-D815, 2013.

24. Bader GD and Hogue CW: An automated method for finding molecular complexes in large protein interaction networks. BMC Bioinformatics 4: 2, 2003.
25. Apweiler R, Attwood TK, Bairoch A, Bateman A, Birney E, Biswas M, Bucher P, Cerutti L, Corpet F, Croning MD, et al: The InterPro database, an integrated documentation resource for protein families, domains and functional sites. Nucleic Acids Res 29: 37-40, 2001.

26. Filipowicz W, Bhattacharyya SN and Sonenberg N: Mechanisms of post-transcriptional regulation by microRNAs: Are the answers in sight? Nat Rev Genet 9: 102-114, 2008.

27. Itesako T, Seki N, Yoshino H, Chiyomaru T, Yamasaki T, Hidaka H, Yonezawa T, Nohata N, Kinoshita T, Nakagawa M and Enokida H: The MicroRNA expression signature of bladder cancer by deep sequencing: The functional significance of the miR-195/497 cluster. PLoS One 9: e84311, 2014.

28. Han Y, Chen J, Zhao X, Liang C, Wang Y, Sun L, Jiang Z, Zhang Z, Yang R, Chen J, et al: MicroRNA expression signatures of bladder cancer revealed by deep sequencing. PLoS One 6: e18286, 2011.

29. McConkey DJ, Lee S, Choi W, Tran M, Majewski T, Lee S, Siefker-Radtke A, Dinney C and Czerniak B: Molecular genetics of bladder cancer: Emerging mechanisms of tumor initiation and progression. Urol Oncol 28: 429-440, 2010.

30. Ueno K, Hirata H, Hinoda Y and Dahiya R: Frizzled homolog proteins, microRNAs and Wnt signaling in cancer. Int J Cancer 132: 1731-1740, 2013.

31. Zou H, Osborn NK, Harrington JJ, Klatt KK, Molina JR, Burgart LJ and Ahlquist DA: Frequent methylation of eyes absent 4 gene in Barrett's esophagus and esophageal adenocarcinoma. Cancer Epidemiol Biomarkers Prev 14: 830-834, 2005.

32. Gibbs GM, Roelants K and O'Bryan MK: The CAP superfamily: Cysteine-rich secretory proteins, antigen 5 , and pathogenesis-related 1 proteins-roles in reproduction, cancer, and immune defense. Endocr Rev 29: 865-897, 2008.

33. Fanjul-Fernández M, Quesada V, Cabanillas R, Cadiñanos J, Fontanil T, Obaya A, Ramsay AJ, Llorente JL, Astudillo A, Cal S and López-Otín C: Cell-cell adhesion genes CTNNA2 and CTNNA3 are tumour suppressors frequently mutated in laryngeal carcinomas. Nat Commun 4: 2531, 2013.

34. Rai A, Menon AV and Jalan S: Randomness and preserved patterns in cancer network. Sci Rep 4: 6368, 2014.

35. He $\mathrm{X}$ and Zhang J: Why do hubs tend to be essential in protein networks? PLoS Genet 2: e88, 2006.

36. Tognon CE and Sorensen PH: Targeting the insulin-like growth factor 1 receptor (IGF1R) signaling pathway for cancer therapy. Expert Opin Ther Targets 16: 33-48, 2012.

37. Tsai TF, Lin YC, Chen HE, Chou KY, Lin JF and Hwang TI: Involvement of the insulin-like growth factor I receptor and its downstream antiapoptotic signaling pathway is revealed by dysregulated microRNAs in bladder carcinoma. Urological Sci 25: 58-54, 2014.

38. Puzio-Kuter AM, Castillo-Martin M, Kinkade CW, Wang X, Shen TH, Matos T, Shen MM, Cordon-Cardo C and Abate-Shen C: Inactivation of p53 and Pten promotes invasive bladder cancer. Genes Dev 23: 675-680, 2009.

39. Berchtold MW and Villalobo A: The many faces of calmodulin in cell proliferation, programmed cell death, autophagy, and cancer. Biochim Biophys Acta 1843: 398-435, 2014.

40. Lopez-Bergami P, Lau E and Ronai Z: Emerging roles of ATF2 and the dynamic AP1 network in cancer. Nat Rev Cancer 10: 65-76, 2010.

41. Manning G, Whyte DB, Martinez R, Hunter T and Sudarsanam S: The protein kinase complement of the human genome. Science 298: 1912-1934, 2002.

42. Roskoski R Jr: RAF protein-serine/threonine kinases: Structure and regulation. Biochem Biophys Res Commun 399: 313-317, 2010.

43. Zebisch A and Troppmair J: Back to the roots: The remarkable RAF oncogene story. Cell Mol Life Sci 63: 1314-1330, 2006.

44. Brault L, Gasser C, Bracher F, Huber K, Knapp S and Schwaller J: PIM serine/threonine kinases in the pathogenesis and therapy of hematologic malignancies and solid cancers. Haematologica 95: 1004-1015, 2010.

45. Roberts PJ and Der CJ: Targeting the Raf-MEK-ERK mitogen-activated protein kinase cascade for the treatment of cancer. Oncogene 26: 3291-3310, 2007.

46. Tao HC, Wang HX, Dai M, Gu CY, Wang Q, Han ZG and Cai B: Targeting SHCBP1 inhibits cell proliferation in human hepatocellular carcinoma cells. Asian Pac J Cancer Prev 14: 5645-5650, 2013.

This work is licensed under a Creative Commons

Attribution-NonCommercial-NoDerivatives 4.0

International (CC BY-NC-ND 4.0) License. 